\title{
Finite Element Analysis of the Generation and Propagation Processes of Electromagnetic Ultrasonic Transverse Wave in stainless steel plate
}

\author{
Xuming Tang ${ }^{1, a}$, Nengjun Yan ${ }^{1, b}$ \\ ${ }^{1}$ Xi'an Institute of High Tech Research, Xi'an 710025, China ; \\ atming26@foxmail.com, byangbcpl@163.com
}

\begin{abstract}
The working process of electromagnetic ultrasonic transducer(EMAT) which involves the intercoupling between electromagnetic field, force field and acoustic field is complex. This paper analyzes the structure and principle of EMAT. And on the basis of it , using COMSOL Multiphysics finite element software to simulate the EMAT, it obtains the distribution characteristics of electromagnetic ultrasonic excitation, electromagnetic field and force field in the process of ultrasonic propagation, providing a theoretical basis for the design and optimization of EMAT.
\end{abstract}

Keywords: Electromagnetic Ultrasonic transduce, Lorenz force, Finite element simulation of COMSOL Multiphysics.

\section{Introduction}

Ultrasonic detection is widely used in pipe and plate detection, but because of the limitation of working mechanism, the traditional piezoelectric ultrasonic transducer is difficult to meet the testing requirements in the rough, high temperature or high speed and other special conditions. The EMAT can be used to produce ultrasonic wave directly in the specimen, which can be used without any coupling agent, and can produce many kinds of ultrasonic wave[1]. The use of EMAT to measure the thickness of sheet metal is an important application of electromagnetic ultrasonic testing.

In recent years, the electromagnetic ultrasonic thickness measurement technology has developed rapidly. In the United States, the use of electromagnetic ultrasonic thickness gauge replaced the piezoelectric ultrasonic thickness gauge in oil and gas, rail, pipeline and other fields of application in ten years ago[2]. In China, Yongqian Li developed high precision hand-held electromagnetic ultrasonic thickness meter based on high power FPGA transmission circuit and low noise receiving circuit[3]; Yihua Kang proposed a simple electromagnetic ultrasonic thickness measuring method[4].

Most of the above research is the development of electromagnetic ultrasonic thickness measurement equipment, but in practice, the efficiency of EMAT is low and the signal attenuation is extrude. Therefore, this paper based on the electromagnetic ultrasonic transverse wave thickness measure, built three-dimensional mode of EMAT by the finite element software COMSOL Multiphysics to simulate and calculate the production and transmission of electromagnetic ultrasonic in stainless steel plate, to provide guidance for structure design and optimization of EMAT..

\section{Thickness measurement principle of electromagnetic ultrasonic transverse wave}

Electromagnetic ultrasonic thickness measurement system is mainly composed of a permanent magnet, a coil and a test piece shown in fig.1 [5]. The alternating current fed to the coil induces eddy currents within the skin depth of the specimen. In the present of a large bias magnetic field, these eddy currents lead to body force at the surface layer of the specimen. These forces lead to the launching of an transverse wave into the specimen. 


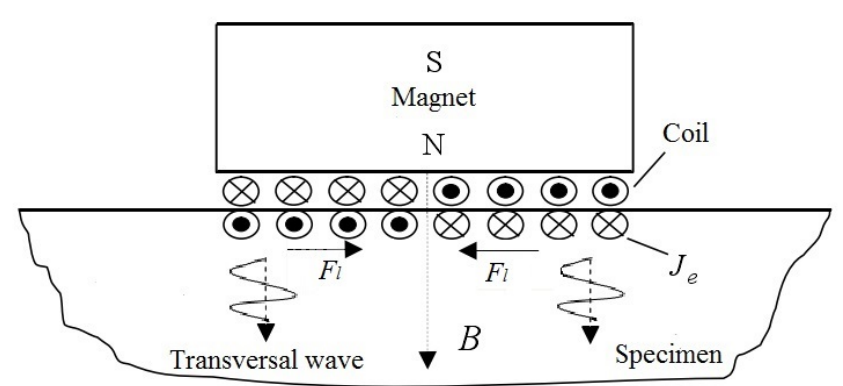

Fig. 1 EMAT configuration

According to the theory of electromagnetic field, the process can be expressed as:

$$
\left\{\begin{array}{l}
\nabla \times \boldsymbol{H}_{d}=\boldsymbol{J}_{c} \\
\boldsymbol{B}_{d}=\mu \boldsymbol{H}_{d} \\
\nabla \times \boldsymbol{E}=-\frac{\partial \boldsymbol{B}_{d}}{\partial \boldsymbol{t}} \\
\boldsymbol{J}_{\boldsymbol{e}}=\varepsilon \boldsymbol{E} \\
\boldsymbol{F}_{l}=\boldsymbol{J} \times\left(\boldsymbol{B}_{d}+\boldsymbol{B}_{s}\right)
\end{array}\right.
$$

Where $\boldsymbol{H}_{d}$ is magnetic field strength, $\boldsymbol{J}_{c}$ is coil current density, $\boldsymbol{B}_{d}$ is magnetic flux, $\boldsymbol{\mu}$ is permeability of specimen, $\boldsymbol{E}$ is electric field intensity, $\boldsymbol{J}_{e}$ is eddy current density, $\varepsilon$ is conductivity, $\boldsymbol{F}_{\boldsymbol{l}}$ is Lorenz force, $\boldsymbol{B}_{s}$ is static magnetic flux.

\section{Research on 3D Modeling of EMAT}

\subsection{EMAT Modeling.}

1) Geometry modeling

Building the geometric modeling of EMAT including coil, magnet, steel plate and air using COMSOL shown in Fig.2. The coil is a racetrack coil which effective length is $20 \mathrm{~mm}$,radius is $0.2 \mathrm{~mm}$, the distance between the conductors is $3 \mathrm{~mm}$, and lift-off is $0.5 \mathrm{~mm}$. The permanent is Nd-Fe-B magnet which size is $25 \mathrm{~mm} \times 25 \mathrm{~mm} \times 15 \mathrm{~mm}(L \times W \times H)$, it's remanence is1.2T. Since the electromagnetic conversion process is mainly concentrated in a limited range below the coil, in order to reduce the amount of calculation, only modeling the main areas of EMAT electromagnetic energy conversion .The size of steel plate is $50 \mathrm{~mm} \times 50 \mathrm{~mm} \times 8 \mathrm{~mm}(L \times W \times H)$,conductivity is $4.032 \times 10^{6} \mathrm{~S} / \mathrm{m}$, density is $7.85 \mathrm{~g} / \mathrm{cm}^{3}$,young modulus is $2 \times 10^{11} \mathrm{~Pa}$. The excitation current is sinusoidal alternating current which amplitude is $50 \mathrm{~A}$, frequency is $500 \mathrm{KHz}$. The coordinate system is shown in Fig.2

2)Physical Field

The physical models of magnetic field and solid mechanics are set up in the model. Analyzing the magnetic ,eddy currents and Lorenz force by the magnetic field module. Studying on particle displacement and ultrasonic wave propagation in steel plate by solid mechanics.

3)Meshing

In order to ensure the accuracy of simulation, the finite element mesh must be fine enough. The finer the mesh subdivision is, the more accurate the results are, but the amount of computation is also increasing rapidly. In order to satisfy the requirements of accuracy and computational results,seting two grid mesh in the skin depth of steel plate where the electromagnetic energy is concentrated and changes sharply. In the rest of the area, the mesh is divided by using the free split tetrahedron. 


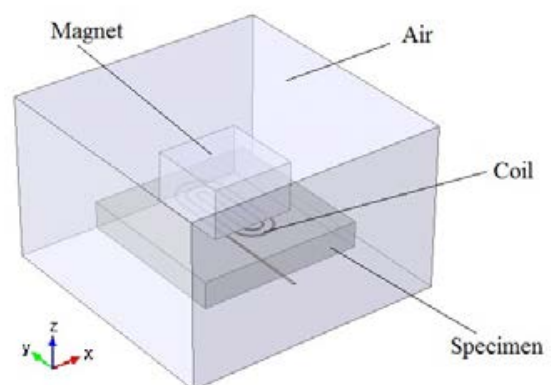

Fig. 2 Geometric model of EMAT

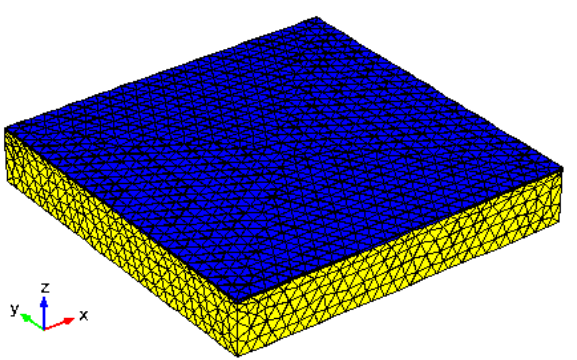

Fig. 3 Meshing of steel plate

\subsection{Simulation Results and Analysis}

1) Analysis of static magnetic flux

Shown in Fig.4 a), The static magnetic field mainly concentrated in the region below the permanent magnet, and is non-uniform. The magnetic field near the edge of the permanent magnet is the strongest, and the maximum value is 0.39 T.Fig. 4 b) show the magnetic flux density distribution in the XOZ section. The direction of the magnetic field in the magnet center is perpendicular to the surface of the steel plate, while the direction of the magnetic field in the magnet edge tends to be horizontal. Therefore, in the previous simulation study, the way to simplify calculation by assuming that the static magnetic field generated by the permanent magnet is uniform will reduce the precision of model.

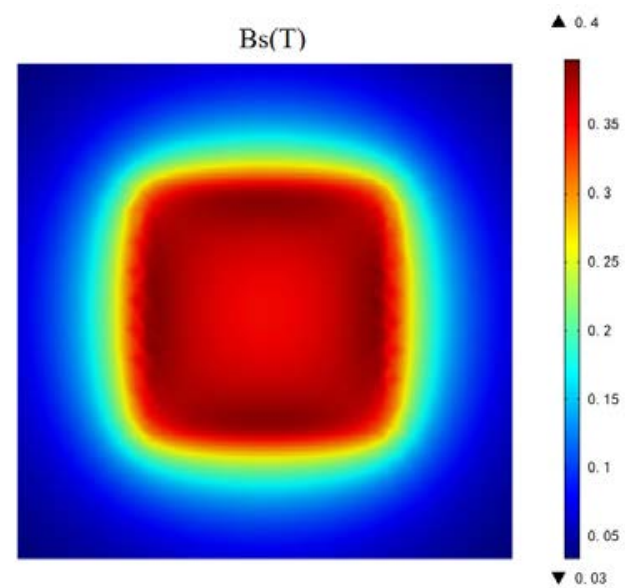

a) surface of steel plate

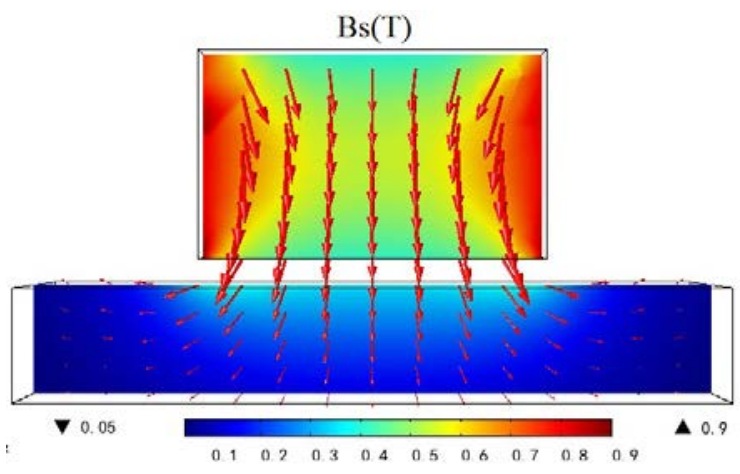

b) XOZ section

Fig. 4 Static magnetic flux density distribution

2)Analysis of eddy current and Lorenz force

Fig. 5 shows the dynamic magnetic flux density distribution at $1.25 \mu \mathrm{s}$. The dynamic magnetic field is mainly concentrated on the lower part of the coil,and its contour is consistent with the shape of coil.The maximum value of dynamic magnetic field is $0.13 \mathrm{~T}$, which is less than that of the static magnetic field,but the both is in one order of magnitude.Therefore, the dynamic magnetic field can not be neglected when analysing the Lorenz force in the steel plate. 


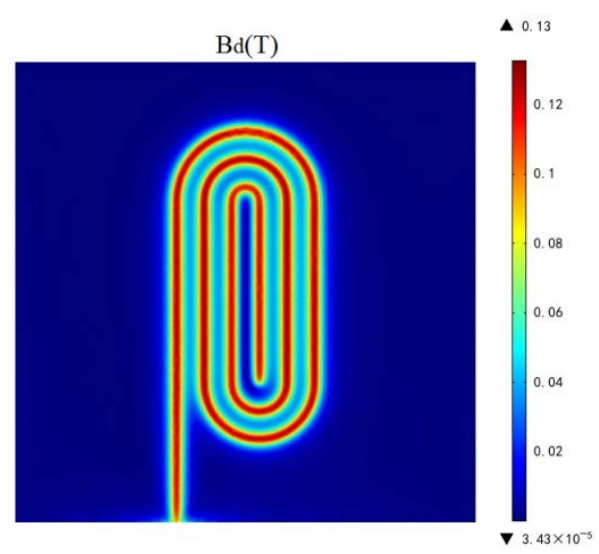

Fig. 5Dynamic magnetic flux density distribution distribution

Y-axis direction of eddy current distribution at $1.25 \mu \mathrm{s}$ is shown in Fig.6. The eddy current in the steel plate is mainly distributed along the contour of the coil, which is similar to the distribution of the dynamic magnetic field, which indicates that the dynamic magnetic field in the steel plate is the main cause of eddy current.

Lorenz force is generated under the action of static magnetic field and dynamic magnetic field within the skin depth of steel plate.The distribution of three direction of the Lorenz force density in the surface of steel plate at $1.25 \mu \mathrm{s}$ is shown in Fig.7 which reflect the characteristics of the distribution and numerical value of the Lorenz force.

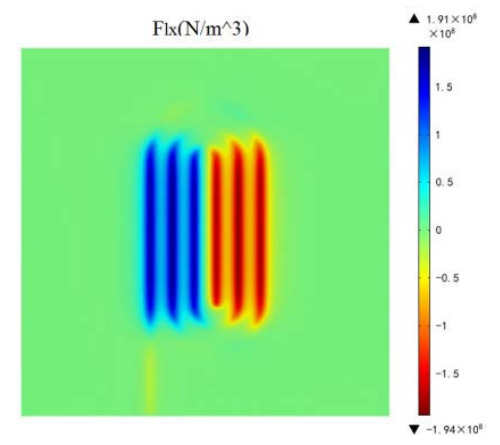

a)X-axis direction

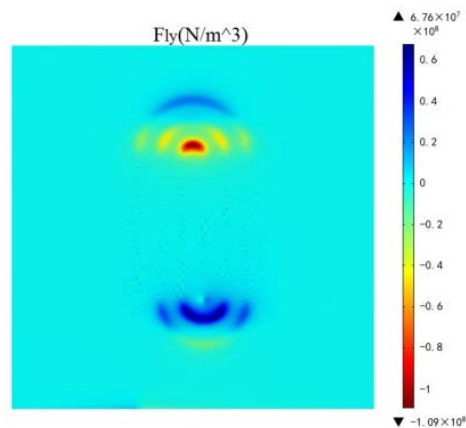

b)Y-axis direction

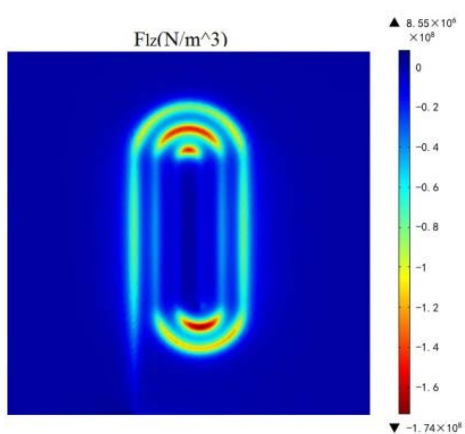

c)Z-axis direction

Fig. 7 Lorenz force density distribution in the surface of steel plate at $1.25 \mu \mathrm{s}$

In the aspect of the distribution,the $\mathrm{X}$-axis direction Lorenz force is mainly distributed below the straight wire of the coil.The Y-axis direction Lorenz force is mainly distributed below the winding wire,which is related to the induced current distribution in the $\mathrm{X}$-axis direction. The Z-axis direction Lorenz force is mainly distributed below the outboard straight wire and the winding wire, this is because the Z-axis direction Lorenz force is generated by horizontal offset magnetic field.And the horizontal offset magnetic field is mainly distributed in the area near the edge of the magnet.

In the aspect of the numerical value, the $\mathrm{X}$-axis direction Lorenz force is more than the $\mathrm{Y}$-axis direction.Therefore, the $\mathrm{X}$-axis direction Lorenz force is the main cause of the propagation of transverse waves along the thickness of the steel plate.The value of Z-axis direction Lorenz force is between the $\mathrm{X}$-axis direction and the $\mathrm{Y}$-axis direction. It mainly produces transverse waves propagating along the surface of the steel plate and longitudinal waves propagating along the thickness of the steel plate.So the size of the magnet and the coil should be considered to reduce the size of the Z-axis direction Lorenz force in the design of the EMAT using transverse waves to measure thickness.

3)Analysis of wave generation and propagation

The Lorenz force on the surface of the steel plate has components in the three axis directions, therefore, the particle on1the surface of the steel plate must have the displacement in the three axis directions. The variation of the average value of the particle displacement component is shown in 
Fig.8. The displacement in the X-axis direction of the particle has obvious advantages in the total displacement.

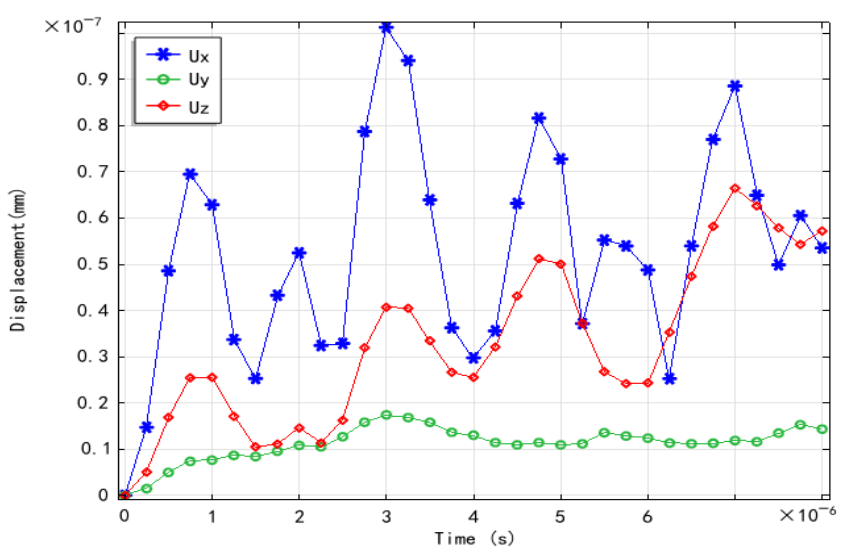

Fig. 8 Variation of the average value of the particle displacement component

In the section of steel plate, defining four points1 4, the coordinate is $\left(-4.5,0, z_{i}\right)$ (where $z_{i}=-6,-8,-10,-12$ ) . Fig.9 shows the variation of displacement of four different points.

The paricles vibrate in the center of the equilibrium position, and the amplitude of the particle decreases rapidly with the increase of the thickness of the steel plate. Particle 1 and particle 3 reach the second positive peaks at $3.72 \mu \mathrm{s}$ and $4.88 \mu \mathrm{s}$, the wave velocity can be calculated as $3448.3 \mathrm{~m} / \mathrm{s}$ which is in accordance with the velocity of transverse wave in steel. The peak values of the second positive peaks of the two particles are $3.88 \times 10^{-8} \mathrm{~mm}$ and $8.29 \times 10^{-8} \mathrm{~mm}$, the amplitude decreased by $78.6 \%$, which show that the electromagnetic ultrasonic has obvious attenuation in the propagation in steel.

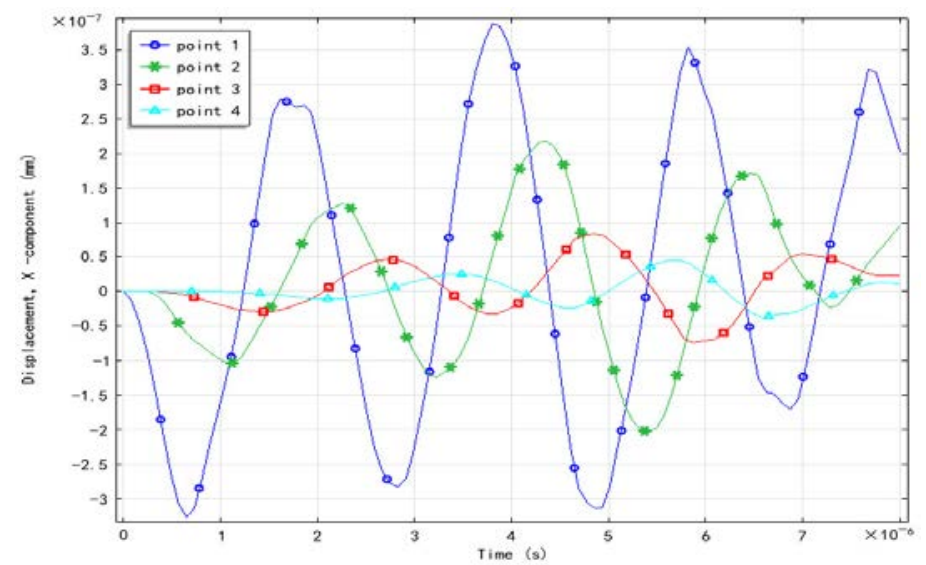

Fig. 9 Variation of displacement of four different points

\section{Conclusion}

(1) Coil with high frequency alternating current can induce dynamic magnetic field in the skin depth of steel plate which which induces the same frequency eddy current, and the current distribution region is consistent with the coil contour.

(2) The static magnetic field excited by the magnet in the steel plate is non-uniform, which mainly focuses on the edge of the magnet. And the magnetic field direction in different regions is different, which makes the Lorenz force in different regions of the steel plate is different in value and direction.

(3) The ultrasonic wave propagating in the thickness direction is the superposition of the transverse wave and longitudinal wave. The transverse wave is the main part, which can be used to measure the thickness of steel plate, but the attenuation is larger. Therefore, in order to stimulate the high-intensity, well-directivity ultrasonic, the parameters of each part of EMAT should be optimized. 


\section{References}

[1]. Lijian Yang, Chunhua Li, Wenping Gao, et al. Finite element simulation of the generation and propagation processes of electromagnetic ultrasonic wave in aluminum plate test. Chinese Journal of Scientific Instrument.33 (2012) 1218-1223.

[2]. ERGANDER M J. EMAT thickness measurement for tubes in coal—fired boilers. Applied Energy.74(2003) 439-444.

[3]. Yongqian Li, Zheng Sun, Hui Zhao,et al. High Accuracy Hand-held Thickness Gauge. Instrument Technique and Sensor.32 (2016) 32-34.

[4]. Yihua Kang, Jun Tu, Yun Yang, et al. Research on the Implementation Methodof a Simple Thickness Measurement Method by Using EMAT. Process Automation Instrumentation. 33(2012) 83-85.

[5]. Junzhe Chen: Research of the Steel Thickness Detection Technology Based on the Electromagnetic Ultrasonic l. Shenyang University of Technology, China ,2015. 